\title{
Cardiac hypertrophy in mice with long-chain acyl-CoA dehydrogenase or very long-chain acyl-CoA dehydrogenase deficiency
}

\author{
Keith B Cox ${ }^{1}$, Jian Liu², Liqun Tian', Stephen Barnes ${ }^{3,4}$, Qinglin Yang ${ }^{2}$ and Philip A Wood ${ }^{1,2,5}$
}

Cardiac hypertrophy is a common finding in human patients with inborn errors of long-chain fatty acid oxidation. Mice with either very long-chain acyl-coenzyme A dehydrogenase deficiency (VLCAD-/-) or long-chain acyl-coenzyme A dehydrogenase deficiency (LCAD-/-) develop cardiac hypertrophy. Cardiac hypertrophy, initially measured using heart/ body weight ratios, was manifested most severely in LCAD-/- male mice. VLCAD-/- mice, as a group, showed a mild increase in normalized cardiac mass (8.8\% hypertrophy compared with all wild-type (WT) mice). In contrast, LCAD-/- mice as a group showed more severe cardiac hypertrophy (32.2\% increase compared with all WT mice). On the basis of a clear male predilection, we analyzed the role of dietary plant estrogenic compounds commonly found in mouse diets because of soy or alfalfa components providing natural phytoestrogens or isoflavones in cardioprotection of LCAD-/- mice.

Male LCAD-/- mice fed an isoflavone-free test diet had more severe cardiac hypertrophy $(58.1 \%$ hypertrophy compared with WT mice fed the same diet). There were no significant differences in the female groups fed any of the diets. Echocardiography measurement performed on male LCAD-deficient mice fed a standard diet at the age of $\sim 3$ months confirmed the substantial cardiac hypertrophy in these mice compared with WT controls. Left ventricular (LV) wall thickness of the interventricular septum and posterior wall was remarkably increased in LCAD-/- mice compared with that of WT controls. Accordingly, the calculated LV mass after normalization to body weight was increased by about $40 \%$ in the LCAD-/- mice compared with WT mice. In summary, we found that metabolic cardiomyopathy, expressed as hypertrophy, developed in mice because of either VLCAD deficiency or LCAD deficiency; however, LCAD deficiency was the most profound and seemed to be attenuated either by endogenous estrogen (in females) or by phytoestrogens present in the diet as isoflavones (in males).

Laboratory Investigation (2009) 89, 1348-1354; doi:10.1038/labinvest.2009.86; published online 7 September 2009

KEYWORDS: long-chain acyl-CoA dehydrogenase; very long-chain acyl-CoA dehydrogenase; cardiac hypertrophy; isoflavones; phytoestrogens

Inborn errors of long-chain fatty acid metabolism are often characterized by cardiac hypertrophy, arrhythmia and sudden death in the disease phenotype ${ }^{1}$ of affected children. These metabolic defects involve disruption of long-chain fatty acid catabolism, either in the transport of long-chain acylcarnitine esters into the mitochondria (as in carnitine palmitoyltransferase-2 or carnitine/acylcarnitine translocase deficiencies) or in long-chain fatty acid $\beta$-oxidation (as in very long-chain acyl-CoA dehydrogenase or long-chain 3-hydroxy-acyl-CoA dehydrogenase deficiencies). In addition, in some of these cases, attenuation of disease severity has been correlated with the presence of residual enzyme activity. ${ }^{2-4}$ The molecular basis of metabolic cardiomyopathy, expressed as hypertrophy, that results from primary defects in metabolism is poorly understood. Because the heart relies heavily on fatty acid oxidation (FAO) for energy production after birth, it has been considered that energy deprivation contributes to the development and progression of metabolic cardiomyopathy. ${ }^{5}$ In addition, certain inborn errors of longchain fatty acid metabolism, as well as ischemia and reperfusion injury, can result in accumulation of toxic long-chain acylcarnitines. These amphipathic molecules are thought to

\footnotetext{
'Department of Genetics, University of Alabama at Birmingham, Birmingham, AL, USA; ${ }^{2}$ Department of Nutrition Sciences, University of Alabama at Birmingham, Birmingham, AL, USA; ${ }^{3}$ Department of Pharmacology and Toxicology, University of Alabama at Birmingham, Birmingham, AL, USA; ${ }^{4}$ Purdue University-University of Alabama at Birmingham Botanicals Center for Age-related Disease, University of Alabama at Birmingham, Birmingham, AL, USA and ${ }^{5}$ Burnham Institute for Medical Research, Orlando, FL, USA

Correspondence: Dr PA Wood, DVM, PhD, Metabolic Signaling \& Disease Program, Diabetes and Obesity Research Center, Burnham Institute for Medical Research at Lake Nona, 6400 Sanger Road, Orlando, FL 32827, USA.

E-mail: pwood@burnham.org
}

Received 24 March 2009; revised 26 June 2009; accepted 29 June 2009 
be arrhythmogenic because they alter membrane properties and integral proteins such as the sarcolemmal $\mathrm{Na}^{+} / \mathrm{K}^{+}$-ATPase. ${ }^{6}$ Long-chain acyl-coenzyme A dehydrogenase (LCAD) ${ }^{7}$ and very long-chain acyl-coenzyme A dehydrogenase $(\mathrm{VLCAD})^{8}$ deficient mouse models have been developed.

It has long been established that hormone status as determined by sex and age has an important role in the development and progression of cardiovascular disease. ${ }^{9}$ More recently, plant-derived estrogen-like compounds, such as the isoflavones, have been cited as agents that can influence the development and course of cardiovascular disease in people who eat a diet rich in certain plant proteins such as those derived from soy. ${ }^{10-15}$ There are no known human patients with LCAD deficiency; however, the LCAD-deficient mouse model, which has a more severe phenotype ${ }^{7,8}$ than the VLCAD-deficient model, has many shared features of human patients with VLCAD deficiency. We hypothesized that the mouse models for VLCAD and LCAD deficiencies would develop cardiac hypertrophy as seen in the corresponding human VLCAD deficiency disease. We initially analyzed cardiac mass in both male and female LCAD-deficient, VLCAD-deficient and wild-type (WT) control mice. We then determined the effects of dietary isoflavones (phytoestrogens) on cardiac mass changes, and finally evaluated heart mass and cardiac function in the C57BL/6 congenic LCAD-deficient mice by echocardiography.

\section{MATERIALS AND METHODS Animals}

All mutant mice in the study were homozygous at either the long-chain acyl-coenzyme A dehydrogenase locus (Acadl) (B6,129-Acadl ${ }^{\text {tmluab }}$ LCAD-/-) $\quad(\mathrm{LCAD}=$ enzyme; Acadlmouse, $A C A D L$-human $=$ genes, respectively $)^{7}$ or the very long-chain acyl-coenzyme A dehydrogenase locus (Acadvl) $\left(\mathrm{B} 6,129-A c a d v l^{\mathrm{tm} 1 \mathrm{Uab}} \quad\right.$ VLCAD-l-) $\quad(\mathrm{VLCAD}=$ enzyme; Acadvl-mouse, $A C A D V L$-human $=$ genes, respectively), ${ }^{8}$ or had B6,129-Acadl ${ }^{+1+}$ and B6,129-Acadvl ${ }^{+1+}$ WT alleles. An equivalent B6, 129 background was maintained by using intercrosses within each of the three genotypes. LCAD-/-, VLCAD-/- and WT mice in the initial studies were derived from mice of 129/SvEvTacBR (129S6) and C57BL/6NTacfBR (B6) (Taconic) genetic backgrounds. Mice used for the echocardiography studies were LCAD-/- B6 congenic mice, all males, $\sim 12$ weeks old, and fed a standard diet (HarlanTeklad 7913-NIH-31). Mice were negative for murine pathogens based on serological assays for 10 different viruses, aerobic bacterial cultures of the nasopharynx and cecum, examinations for endo- and ectoparasites, and histopathology of all major organs. All animal protocols were approved by the Institutional Animal Care and Use Committee of the University of Alabama at Birmingham.

\section{Heart Mass Measurement}

Before killing and sample collection, all mice except those in the diet studies were fasted for $22-28 \mathrm{~h}$. Following measure- ment of body weight, hearts were carefully excised and gently blotted on absorbent paper to remove blood from the ventricles before wet weight measurement.

\section{Echocardiography Measurement}

M-mode echocardiography was performed using the small animal echocardiography analysis system (Vevo 770, VisualSonics, Canada). In brief, mice were anesthetized with isoflurane inhalation in oxygen. Fur was removed from the upper sternal and subxiphoid areas. Limbs were attached to electrodes for electrocardiographic monitoring and timing of cardiac cycles. A two-dimensional short-axis view and M-mode tracings of the left ventricle were obtained with a $30 \mathrm{MHz}$ transducer. Using analysis software provided by the manufacturer, the following data were obtained: left ventricular dimension at systole and diastole (LVID;s and LVID;d), posterior wall thickness at systole and diastole (LVPW;s and LVPW;d), interventricular septal wall thickness at systole and diastole) (IVS;s and IVS; d:), ejection fraction (EF\%), fractional shortening (FS\%), left ventricle volume at systole and diastole (volume;s and volume; d), and left ventricle stroke volume, heart rate, cardiac output and left ventricular mass.

\section{Diet Study Design}

At 21 days of age LCAD- $/$ - and LCAD $+/+$ mice were weaned onto either a standard laboratory diet (Teklad LM-485-the basal diet) or an isoflavone-free diet (Teklad diet TD.96155-the test diet) (Harlan-Teklad, Inc., Indianapolis, IN, USA). Mice were housed under 12-h lightdark cycles in microisolator cages and killed at 90-115 days of age for sample collection. The mice in the diet study were killed by $\mathrm{CO}_{2}$ inhalation and the body and wet heart weight measurements were taken as above, but without prior fasting.

\section{Measurement of Isoflavones in Diets}

Individual samples $(0.5 \mathrm{~g})$ of each diet were analyzed in duplicate. The isoflavones were extracted by mixing with $80 \%$ aqueous methanol $(5 \mathrm{ml})$ at $4^{\circ} \mathrm{C}$ for $2 \mathrm{~h}$. Dichlorofluorescein was added as an internal standard to the extracting solvent. ${ }^{16}$ After centrifugation of the mixture at $3000 \mathrm{~g}$ for $10 \mathrm{~min}$, the supernatants were analyzed using reverse-phase HPLC on an Agilent Technologies HP 1100 instrument. Aliquots $(10 \mu \mathrm{l})$ were separated on a $22 \mathrm{~cm} \times$ $4.6 \mathrm{~mm}$ i.d. Aquapore $\mathrm{C}_{8}$ column with a $3 \mathrm{~cm} \times 4.6 \mathrm{~mm}$ i.d. $\mathrm{C}_{8}$ guard column. The column was equilibrated in solvent A $(90 \%$ double-distilled water: $10 \%$ acetonitrile containing $0.1 \%$ trifluoroacetic acid). The isoflavones were eluted with a $0-30 \%$ gradient of solvent B $(10 \%$ double-distilled water:90\% acetonitrile containing $0.1 \%$ trifluoroacetic acid) in solvent $\mathrm{A}$ over a 30 -min period at a flow rate of $1.5 \mathrm{ml} / \mathrm{min}$. Eluted isoflavones were monitored with a diode array detector and assayed from their absorbance at $262 \mathrm{~nm}$. Standard isoflavone mixtures also containing the internal standard were analyzed as part of the group of samples. 
Quantitative data were obtained by comparing the ratios of the areas of the unknown peaks to the internal standard with those in the standard isoflavone mixtures. Results are reported in aglucone units, that is, the equivalent weight of the isoflavone aglucone regardless of the nature of glucose moiety (the isoflavones are present in soy as at least three $\beta$-glucoside conjugates, each with a different molecular weight). Within-assay variation for duplicate samples ranged from 1 to $3 \%$ and inter-assay variation from 3 to $5 \%$.

\section{Statistics}

Groups were analyzed by either one-way or two-way analysis of variance (ANOVA) using the SigmaStat 2.03 as program parameters permitted.

\section{RESULTS \\ Heart Weight to Body Weight Ratio in LCAD- and VLCAD-Deficient Mice}

Figure 1 shows the effects of genotype, sex and age on cardiac mass normalized to body weight. Genotype had the greatest effect on the development of cardiac hypertrophy $(P<0.001)$. The VLCAD-/- mice had a milder cardiac phenotype. VLCAD-/- mice, as a group, showed a mild increase in normalized cardiac mass ( $8.8 \%$ hypertrophy compared with all WT mice). In contrast, LCAD-/- mice as a group showed more severe cardiac hypertrophy $(32.2 \%$ increase compared with all WT mice). A closer examination of cardiac mass in subgroups divided along genotype, age and sex lines showed

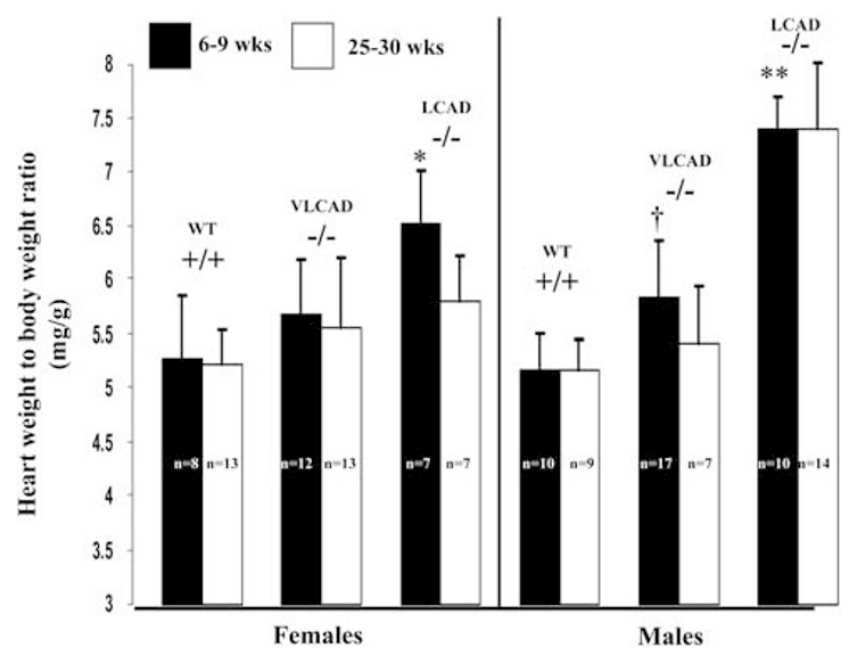

Figure 1 Heart weight to body weight ratios in younger (6- to 9-week old) and older (25- to 30-week old) male and female LCAD-/-, VLCAD-/- and WT mice. Error bars equal 1 s.d.. LCAD-/- male $\left.{ }^{(*}\right)$ and young LCAD-/female $\left({ }^{*}\right)$ mice showed significant cardiac hypertrophy compared with VLCAD-/- and WT mice of like sex and LCAD-/- males $\left(^{* *}\right)$ showed significantly greater hypertrophy than all other female groups $(P<0.001$, one-way ANOVA). Younger VLCAD-/- males ( $\dagger$ ) showed significant hypertrophy compared with age-matched WT males $(P=0.032)$ but older age-matched VLCAD-/- and WT males did not. In contrast with young LCAD-/- females, VLCAD-/- females did not show significant hypertrophy compared with age-matched WT females. that cardiac hypertrophy was less severe in females and certain groups of older mice with either LCAD or VLCAD deficiency, as detailed below.

Sex was the next most influential modulator of cardiac mass $(P<0.001)$. Although sex had no effect on cardiac mass in WT mice, cardiac hypertrophy was more severe in both VLCAD-/- and LCAD-/- males than in females of like genotype. Sex and genotype interacted significantly $(P<0.001)$. Even in younger VLCAD- - males, when cardiac hypertrophy was most prominent, the sex disparity in cardiac mass was greater in LCAD-/- mice (13\% difference between the sexes) than in VLCAD-/- mice (3\% difference).

Age was significant, but least influential $(P<0.02)$. Mice were divided into young (6-10 weeks old) and adult mice (15-44 weeks old). Some groups of mice showed a modest decrease in cardiac hypertrophy in adults. The greatest attenuation in cardiac hypertrophy, toward the normal range, was seen in older, female LCAD-/- mice (a 12\% decrease when compared with young LCAD-/- females). Older VLCAD-/- males also showed regression in cardiac hypertrophy back to the normal range. No age effect on hypertrophy was seen in male LCAD-/- mice. When VLCAD-/mice were excluded from the analysis, the age effect on cardiac hypertrophy was not significant $(P=0.06)$.

\section{Diet Effects on the Severity of Metabolic Cardiomyopathy in LCAD-Deficient Mice}

Recent studies have associated high dietary intake of isoflavones, especially those found in soy proteins, with resistance to human cardiovascular disease. ${ }^{10}$ Because soy and alfalfa are used as protein sources in the standard autoclavable rodent diet that we fed our colony, we considered that the diet might be rich in isoflavones and other phytoestrogens. We hypothesized that these compounds may have a sparing effect on the severity of the cardiac hypertrophy phenotype in LCAD-/- mice, especially males, by providing a 'feminizing' effect, much like the endogenous estrogen of LCAD-/- females.

We designed a feeding trial experiment to test this hypothesis. Most striking was the effect of the isoflavone-free test diet on cardiac hypertrophy in male LCAD-/- mice (Figure 2). No differences were detected between any of the female groups. As expected, 32.4\% hypertrophy was observed in LCAD-/- males on the basal soy-based diet compared with WT males fed the same basal diet $(P<0.001)$. Cardiac hypertrophy, however, was exacerbated in LCAD-/- males fed the isoflavone-free test diet, with $58.1 \%$ hypertrophy as compared with WT males on the same test diet $(P<0.001)$. The difference between the LCAD-/- males fed the basal or test diets was also significant $(P<0.01)$. The test diet had no significant effect on cardiac mass in WT mice.

Male LCAD-/- mice fed the isoflavone-free test diet were significantly smaller than WT males on the test diet $(P<0.01)$ and male LCAD-/- mice on the basal diet $(P<0.001$, Figure 3$)$. The difference between LCAD-/- males 


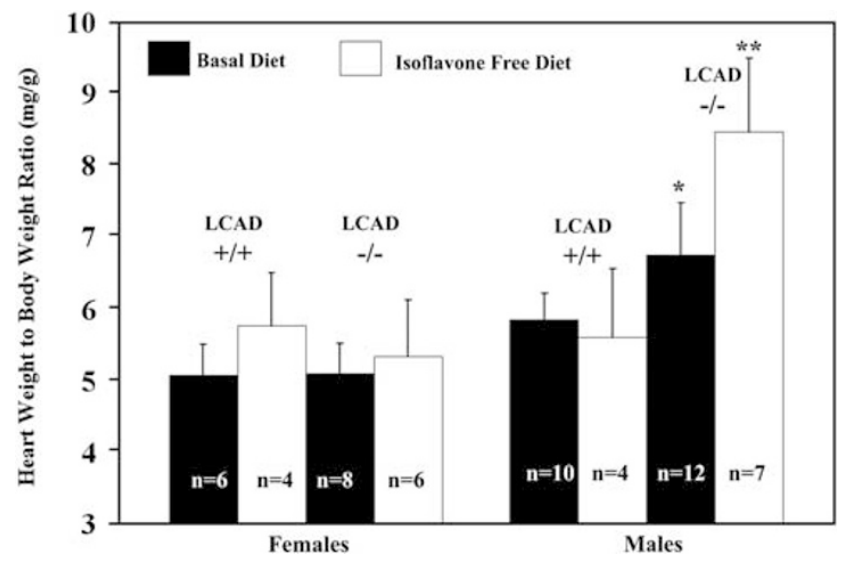

Figure 2 Diet effects on heart weight to body weight ratio in LCAD-/-) mice. Male LCAD-/- mice weaned onto and maintained on an isoflavonefree (test diet) $\left(^{* *}\right)$ showed significantly greater hypertrophy than LCAD-/mice weaned onto the soy containing basal diet or LCAD $+/+(P<0.005$, one-way ANOVA) or LCAD $+/+$ mice on either diet $(P<0.001$, one-way ANOVA). LCAD-/- mice weaned onto the basal diet $\left({ }^{*}\right)$ showed significant hypertrophy compared with WT mice on either diet $(P<0.001$, one-way ANOVA). No differences in heart weight to body weight ratio were noted between WT males on different diets and among any female groups.

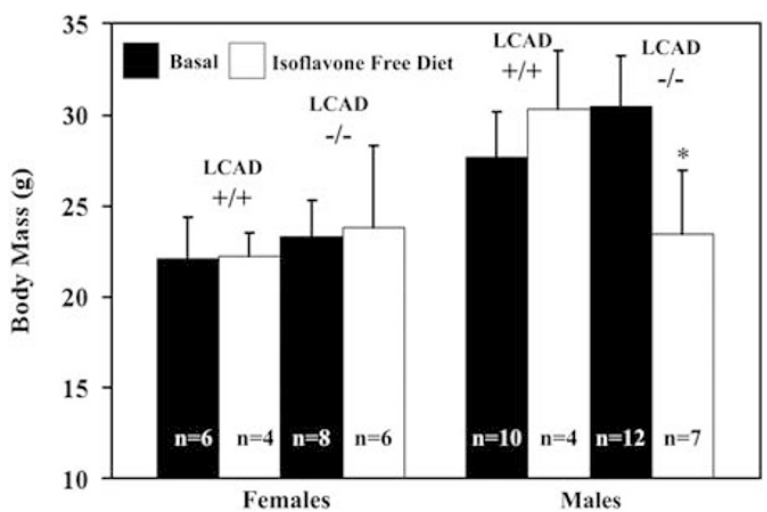

Figure 3 Body mass differences between LCAD-/- and LCAD $+/+$ mice. Body mass was measured in LCAD-/- and WT mice fed either the basal diet or an isoflavone-free test diet. No differences were detected in body mass between any female groups. LCAD-/- male mice fed test diet were significantly smaller than LCAD-/- mice fed the basal diet and the WT males fed the test diet $(P<0.01)$.

on the test diet and WT males on the standard diet was not significant $(P=0.065)$. No detectable effects of diet on weight gain were noted between WT groups of the same sex. In addition, no differences in weight gain were noted among any of the female groups on either diet.

To define the isoflavone content of the two diets used in the diet study, the isoflavone content of each feed was measured (Table 1). The patterns of the isoflavone conjugates (no malonylglucosides and a large proportion of acetylglucosides) were consistent with the use of toasted soy protein in the diet. Total isoflavone concentration implied that the soy protein constituted about $5 \%$ by weight of the total diet.
Table 1 Isoflavone analyses of basal and test (soy-free) diets

\begin{tabular}{lll}
\hline Isoflavone & $\begin{array}{c}\text { Basal diet }(\boldsymbol{\mu} \mathbf{g} / \mathrm{g} \text { diet }) \\
\text { mean } \pm 1 \text { s.d. }\end{array}$ & Test diet \\
\hline Daidzin & $54.3 \pm 2.28$ & 0.00 \\
Glycitin & $8.50 \pm 0.12$ & 0.00 \\
Genistin & $51.5 \pm 3.70$ & 0.00 \\
Malonyl-daidzein & 0.00 & 0.00 \\
Malonyl-genistein & 0.00 & 0.00 \\
Malonyl-glycitein & 0.00 & 0.00 \\
Acetyl-daidzein & $24.8 \pm 1.14$ & 0.00 \\
Acetyl-glycitein & $7.08 \pm 0.14$ & 0.00 \\
Acetyl-genistein & $16.1 \pm 1.07$ & 0.00 \\
Daidzein & 0.00 & 0.00 \\
Glycitein & 0.00 & 0.00 \\
Genistein & 0.00 & 0.00 \\
Total & $162 \pm 8.46$ & 0.00
\end{tabular}

In order to further quantify the original observation of cardiac hypertrophy based on heart-to-body weight ratios, we performed echocardiography studies on later groups of mice. Echocardiography measurement performed on male LCAD-deficient mice at the age of $\sim 3$ months confirmed the substantial cardiac hypertrophy in these mice compared with WT controls (Figure 4, representative M-mode image of the hearts from a LCAD-/- and a WT mice). The left ventricular wall thickness of the interventricular septum and posterior wall was remarkably increased in LCAD-/- mice compared with that of control mice (Table 2). Accordingly, the calculated LV mass after normalization to body weight was increased by about $40 \%$ in the LCAD-/- mice compared with WT mice (Table 2 and Figures 4 and 5). Interestingly, cardiac performance appeared to be well maintained with normal $\mathrm{EF} \%, \mathrm{FS} \%$ and cardiac output (Table 2). Heart rate in the LCAD-/- mice was also comparable with that in WT mice (Table 2).

\section{DISCUSSION}

We propose the LCAD-deficient mice as a model of the metabolically induced cardiomyopathy seen in children with severe VLCAD deficiency. Children born with severe deficiencies in long-chain FAO usually present in metabolic crisis before 6 months of age and episodes of metabolic crisis pose serious threats to survival. ${ }^{17}$ One group reported successful treatment of a VLCAD-deficient patient with medium-chain triglyceride therapy. ${ }^{18}$ Another approach of therapy for cardiomyopathy in human VLCAD deficiency was administration of the anaplerotic, odd-chain fatty acid containing triglyceride and triheptanoin. ${ }^{19}$ No human cases of LCAD deficiency have been documented. Earlier reported cases of human LCAD deficiency have since been reclassified as 

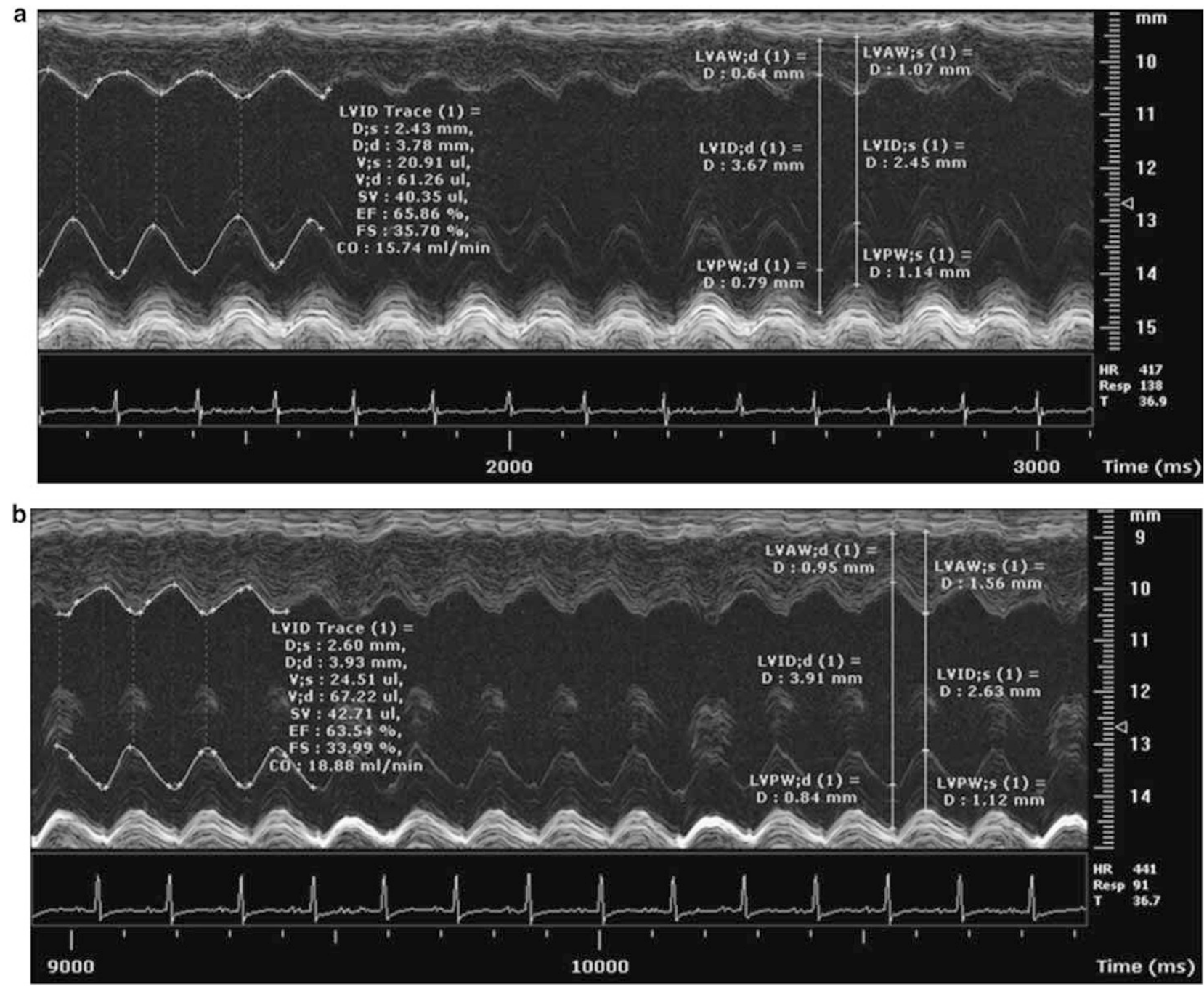

Figure 4 Representative M-mode image of the hearts from a wild-type (WT) (a) and an LCAD-/- (b) mice. Anatomic M-mode (AM-Mode) images obtained from short-axis measurements were used to evaluate left ventricular function (described in Materials and methods). The M-mode spectrum was acquired in WT or LCAD-/- mice at 3 months of age. IVS; s and IVS; $d$, interventricular septal wall thickness (diastole and systole); LVPW; d and LVPW; s, left ventricular posterior wall thickness (diastole and systole); LVID;d and $D ; d$, left ventricular internal diameter (diastole); LVID;s and D;s, left ventricular internal diameter (systole); V;s and V;d, volume; systole and diastole; SV: stroke volume; EF: ejection fraction; FS: fractional shortening; CO: cardiac output. Three to five cardiac cycles were averaged for each experimental animal ( $n=9$ mice for each genotype group) for every measurement for each time point.

VLCAD deficiency. ${ }^{20}$ We also have developed VLCADdeficient mice and they also develop metabolic disease, such as cardiac hypertrophy, albeit milder than that seen in LCADdeficient mice. ${ }^{8}$ The cardiac hypertrophy seen in mice with LCAD deficiency is comparatively milder than the cardiac hypertrophy associated with VLCAD deficiency in human beings. Reasons for this disparity may arise from a diminished reliance on fatty acid $\beta$-oxidation for cardiac energy homeostasis in mice, from a greater substrate overlap between LCAD and VLCAD activities in mice, or from a greater resistance in mice to the effects of toxic metabolites.

Although cardiac hypertrophy in the LCAD-/- mice was quite remarkable compared with many mouse models with cardiac defects, it is interesting to observe that cardiac performance of the LCAD-/- mice was well maintained at least for the ages analyzed. Furthermore, left ventricular dimension was not changed despite the increasing thickness of the left ventricular wall. This is similar to a pattern of concentric cardiac hypertrophy seen in patients with left ventricular pressure overload. Therefore, the cardiac hypertrophy manifested in the LCAD-/- mice represents a typical compensatory hypertrophy. It is not clear yet whether any physiological and/or pathological stress would lead to the development of decompensatory hypertrophy or even progress to heart failure. It is interesting that at least aging, as studied here, did not exacerbate the hypertrophy progression.

As we report here, variation in more chronic features of cardiac hypertrophy can be found as well, eg, the mild cardiac 
Table 2 Echocardiography measurements

\begin{tabular}{lccc}
\hline Parameter & WT & LCAD-l- & $P$ \\
\hline LVID;d (mm) & $3.53 \pm 0.08$ & $3.73 \pm 0.1$ & $>0.05$ \\
LVID;s (mm) & $2.24 \pm 0.09$ & $2.43 \pm 0.15$ & $>0.05$ \\
LVPW;d (mm) & $0.71 \pm 0.04$ & $0.9 \pm 0.06$ & $*<0.01$ \\
LVPW; (mm) & $1.14 \pm 0.05$ & $1.34 \pm 10.08$ & $*<0.01$ \\
IVS;d (mm) & $0.75 \pm 0.01$ & $0.99 \pm 0.03$ & $*<0.01$ \\
IVS;S (mm) & $1.18 \pm 0.04$ & $1.36 \pm 0.05$ & $* 0.05$ \\
EF (\%) & $72 \pm 2.1$ & $69 \pm 2.9$ & $>0.05$ \\
FS (\%) & $41 \pm 1.8$ & $38 \pm 2.4$ & $>0.05$ \\
Volume;d ( $\mu$ l) & $56 \pm 2.7$ & $63 \pm 3.4$ & $>0.05$ \\
Volume;s $(\mu l)$ & $15.9 \pm 1.6$ & $20.5 \pm 2.7$ & $>0.05$ \\
SV $(\mu l)$ & $40 \pm 1.8$ & $42.7 \pm 0.9$ & $>0.05$ \\
Heart rate (BPM) & $420 \pm 25$ & $428 \pm 16$ & $>0.05$ \\
CO (ml/min) & $18 \pm 0.7$ & $18.8 \pm 0.57$ & $>0.05$ \\
LV mass (mg) & $68.7 \pm 4.7$ & $112.2 \pm 7.3$ & $* 0.01$ \\
\hline
\end{tabular}

Abbreviations: LVID;s and LVID;d, left ventricular dimension at systole and diastole; LVPW;s and LVPW;d, posterior wall thickness at systole and diastole; IVS; and IVS; d, interventricular septal wall thickness (diastole and systole); EF\%, Ejection fraction; FS\%, fractional shortening; volume;s and volume;d, left ventricle volume at systole and diastole; SV, left ventricle stroke volume; BPM, beats per minute; $\mathrm{CO}$, cardiac output.

Data were expressed as mean \pm s.e.m., ${ }^{\star} P<0.05, n=9$ in each group.

hypertrophy of VLCAD-/- male mice that resolves with age contrasts with the more severe hypertrophy of LCAD-/males that persists well into adulthood. Furthermore, a protective effect against cardiac hypertrophy was documented in both VLCAD-/- and LCAD-/- females, with the greater effect in the latter. Whether the females benefit more from the presence of higher estrogenic hormones or from the absence of androgenic hormones remains to be proven. If the effect results from the presence of estrogens, then we would predict cardiac hypertrophy to be more severe in females after reproductive senescence.

The interaction between hormone status and the development of cardiac disease is seen not only in the different cardiac disease rates between men and premenopausal women, but also in the rapid rise in heart disease rates seen in postmenopausal women. ${ }^{21}$ These studies show the protective effects of estrogenic hormones in the setting of metabolic disease. The plasticity of the hypertrophic response evident in LCAD-/- mice suggests that the hypertrophic response, even an insult as specific as a metabolic enzyme deficiency, is likely the result of complex processes involving signaling pathways that overlap with those downstream of the estrogen receptor. Another possibility is that the gene expression patterns in response to estrogenic compounds yield a myocardium resistant to metabolic insults.

Perhaps most surprising is the demonstrable benefits of a diet rich in soy isoflavones to male LCAD-/- mice.

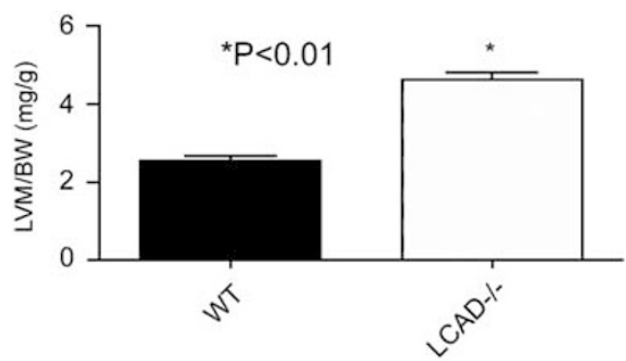

Figure 5 Left ventricular mass to body weight ratio: Left ventricular mass (LVM) of LCAD-/- and WT male mice at their ages of 3 month were derived from M-mode echocardiographic measurements. The resultant LVM was normalized to body weight. Data were expressed as mean \pm s.e.m., $n=9$, $* P<0.01$.

In addition, we found reduced body weight (Figure 3 ) in the group with the most severe cardiac hypertrophy, the LCAD-/- male mice eating the phytoestrogen-free test diet. We speculate that the two effects are not linked. It is noteworthy that this degree of hypertrophy was achieved on a low-fat diet ( $5 \%$ by weight) and that the mice were not challenged with fasting or exercise, conditions that favor the oxidation of fatty acids in the heart. Although a simpler design might have been to add back one or more purified isoflavones to the isoflavone-free diet, a comparison of the measurable isoflavones in each of the diets illustrates the complexity of phytoestrogen composition in standard diet. Of the isoflavones present in the laboratory diet, genistein, binds to the estrogen -receptor with an affinity comparable to that of $17 \beta$-estradiol. ${ }^{22}$ The -glucosides daidzin, genistin and glycitin and their corresponding $6^{\prime}$-O-acetyl esters are hydrolyzed and the resulting aglucones absorbed in the intestine both proximally and distally; they are also metabolized further to equol and other bioactive compounds that are also absorbed. ${ }^{23}$ As a result of this variability in absorption kinetics and because several isoflavone compounds are present in the standard laboratory diet, it is difficult to ascertain which compound or combination of compounds produces attenuation of cardiac hypertrophy in LCAD-/- males. We speculate that the isoflavones are modulating cardiomyocyte cell signaling through activation of the estrogen $\beta$ receptor as described for other organ systems. ${ }^{22}$ The LCAD-/- mice are severely cold intolerant; however, we showed previously that soy-based, isoflavonerich diets make LCAD-/- mice more cold resistant as compared with those fed a casein-isoflavone-free diet. ${ }^{24}$ In contrast, the positive attributes of isoflavone-rich diets are not universal, as in other studies this type of diet was detrimental to mice with a mutation in the $\alpha$-myosin heavy chain gene in which the male mice developed severe, dilated cardiomyopathy when fed a soy-based diet. ${ }^{25}$

Cardiac hypertrophy associated with primary defects in FAO has been considered, at least in part, to be a disease arising out of inadequate energy production. ${ }^{19}$ This was supported by the observation that in adult human beings the 
majority of cardiac energy production is derived from longchain fatty acid $\beta$-oxidation under fasting conditions or during sustained exercise. ${ }^{26}$ Furthermore, children born with 'downstream' deficiencies of medium- and short-chain fatty acid $\beta$-oxidation do not develop cardiac hypertrophy, presumably because they can partially oxidize long-chain fatty acids and derive energy production from the pathway. ${ }^{5}$ An alternate hypothesis is that the formation of long-chain fatty esters such as long-chain acylcarnitine or long-chain acylCoA stimulates the development of cardiac hypertrophy. Studies using rats treated with etomoxir showed that blocking long-chain fatty acid $\beta$-oxidation by preventing the entrance of long-chain fatty acid substrates into the mitochondria is sufficient to evoke a hypertrophic response. ${ }^{27}$ Another possibility is that the more robust hypertrophy seen in LCAD-/- mice results more from abnormal metabolite effects than from interruption of energy production by longchain fatty acids. The similarity of response among male and female mice to etomoxir treatment suggests that inhibition of FAO at the level of CPT1 differs from that seen in LCAD-/and VLCAD- - mice $^{8}$ in that it is not influenced by estrogenmodulated effects.

These studies show the complexity underlying what has been characterized as the programmed, stereotypic response of cardiac hypertrophy. We expect that further investigation of these models will reveal more facets of the interplay between energy metabolism, gene regulation and the hormonal milieu that underlie metabolic cardiomyopathy.

\section{ACKNOWLEDGEMENTS}

We thank Michelle Smith for assistance with the isoflavone analyses. The project described was supported by the National Institutes of Health $(\mathrm{NIH})$ grants RO1-RR02599 (PAW) and training grant T-32-RR00493 (KBC) from the National Center for Research Resources (NCRR), R01-HL084456 (QY) from the National Heart, Lung and Blood Institute (NHLBI) and P50 AT00477 from the National Center for Complementary and Alternative Medicine (NCCAM) and the Office of Dietary Supplements (ODS) to the Purdue University-University of Alabama at Birmingham Botanicals Center for Age-Related Disease (Connie Weaver, PI). The contents of this paper are solely the responsibility of the authors and do not necessarily represent the official views of NCRR, NHLBI, NCCAM, ODS or NIH.

\section{DISCLOSURE/CONFLICT OF INTEREST}

The authors declare no conflict of interest.

1. Rinaldo P, Matern D, Bennett MJ. Fatty acid oxidation disorders. Annu Rev Physiol 2002;64:477-502.

2. Andresen BS, Olpin S, Poorthuis BJ, et al. Clear correlation of genotype with disease phenotype in very-long-chain acyl-CoA dehydrogenase deficiency. Am J Hum Genet 1999;642:479-494.

3. Bonnefont JP, Taroni F, Cavadini P, et al. Molecular analysis of carnitine palmitoyltransferase II deficiency with hepatocardiomuscular expression. Am J Hum Genet 1996;585:971-978.

4. Pande SV. Carnitine-acylcarnitine translocase deficiency. Am J Med Sci 1999:3181:22-27.
5. Roe CR. Diagnostic approach to disorders of fat oxidation. Int Pediatr 1996;11:109-113.

6. Corr PB, Yamada KA. Selected metabolic alterations in the ischemic heart and their contributions to arrhythmogenesis. Herz 1995;203: 156-168.

7. Kurtz DM, Rinaldo $P$, Rhead WJ, et al. Targeted disruption of mouse long-chain acyl-CoA dehydrogenase gene reveals crucial roles for fatty acid oxidation. Proc Natl Acad Sci USA 1998;9526:15592-15597.

8. Cox KB, Hamm DA, Millington DS, et al. Gestational, pathologic and biochemical differences between very long-chain acyl-CoA dehydrogenase deficiency and long-chain acyl-CoA dehydrogenase deficiency in the mouse. Hum Mol Genet 2001;1019:2069-2077.

9. Barrett-Connor E, Grady D. Hormone replacement therapy, heart disease, and other considerations. Annu Rev Public Health 1998; 19:55-72.

10. Burke GL, Vitolins MZ, Bland D. Soybean isoflavones as an alternative to traditional hormone replacement therapy: are we there yet? J Nutr 2000;1303:664S-665S.

11. Lissin LW, Cooke JP. Phytoestrogens and cardiovascular health. J Am Coll Cardiol 2000;356:1403-1410.

12. Martin D, Song J, Mark C, et al. Understanding the cardiovascular actions of soy isoflavones: potential novel targets for antihypertensive drug development. Cardiovasc Hematol Disord Drug Targets 2008;84:297-312.

13. Mann GE, Bonacasa B, Ishii T, et al. Targeting the redox sensitive Nrf2Keap 1 defense pathway in cardiovascular disease: protection afforded by dietary isoflavones. Curr Opin Pharmacol 2009;9:139-145.

14. Carlson S, Peng N, Prasain JK, et al. Effects of botanical dietary supplements on cardiovascular, cognitive, and metabolic function in males and females. Gend Med 2008;5(Suppl A):S76-S90.

15. Wenzel U, Fuchs D, Daniel H. Protective effects of soy-isoflavones in cardiovascular disease. Identification of molecular targets. Hamostaseologie 2008;28:85-88.

16. Coward L, Smith M, Kirk M, et al. Chemical modification of isoflavones in soyfoods during cooking and processing. Am J Clin Nutr 1998;686(Suppl):1486S-1491S.

17. Roe $C R$, Ding J. Mitochondrial fatty acid oxidation disorders. In: Scriver CR, Beaudet AL, Sly WS, Valle D (eds). The Metabolic and Molecular Bases of Inherited Disease, 8th edn. McGraw-Hill: New York, 2001, pp 2297-2326.

18. Brown-Harrison MC, Nada MA, Sprecher $\mathrm{H}$, et al. Very long chain acylCoA dehydrogenase deficiency: successful treatment of acute cardiomyopathy. Biochem Mol Med 1996;581:59-65.

19. Roe CR, Sweetman L, Roe DS, et al. Treatment of cardiomyopathy and rhabdomyolysis in long-chain fat oxidation disorders using an anaplerotic odd-chain triglyceride. J Clin Invest 2002;1102:259-269.

20. Yamaguchi S, Indo Y, Coates PM, et al. Identification of very-long-chain acyl-CoA dehydrogenase deficiency in three patients previously diagnosed with long-chain acyl-CoA dehydrogenase deficiency. Pediatr Res 1993;341:111-113.

21. Barrett-Connor E, Bush TL. Estrogen and coronary heart disease in women. JAMA 1991;26514:1861-1867.

22. Kuiper GG, Lemmen JG, Carlsson B, et al. Interaction of estrogenic chemicals and phytoestrogens with estrogen receptor beta. Endocrinology 1998;13910:4252-4263.

23. Setchell KD, Cassidy A. Dietary isoflavones: biological effects and relevance to human health. J Nutr 1999;1293:758S-767S.

24. Schuler AM, Barnes S, Gower BA, et al. Dietary phytoestrogens increase metabolic resistance (cold tolerance) in long-chain acyl-CoA dehydrogenase-deficient mice. J Nutr 2004;1345:1028-1031.

25. Stauffer BL, Konhilas JP, Luczak ED, et al. Soy diet worsens heart disease in mice. J Clin Invest 2006;1161:209-216.

26. Ascuitto RJ, Ross-Ascuitto NT. Substrate metabolism in the developing heart. Semin Perinatol 1996;206:542-563.

27. Zarain-Herzberg A, Rupp $H$, Elimban V, et al. Modification of sarcoplasmic reticulum gene expression in pressure overload cardiac hypertrophy by etomoxir. FASEB J 1996;1011:1303-1309. 